\title{
Weather Impact on Airport Arrival Meter Fix Throughput
}

\author{
Yao Wang, NASA Ames Research Center, Moffett Field, California
}

\begin{abstract}
Time-based flow management provides arrival aircraft schedules based on arrival airport conditions, airport capacity, required spacing, and weather conditions. In order to meet a scheduled time at which aircraft can cross an airport arrival meter fix prior to entering the airport terminal airspace, air traffic controllers impose regulations on air traffic. Severe weather may create an airport arrival bottleneck if one or more of airport arrival meter fixes are partially or completely blocked by the weather and the arrival demand has not been reduced accordingly. Under these conditions, aircraft are frequently put in holding patterns until they can be rerouted. A model that predicts the weather-impacted meter fix throughput may help air traffic controllers direct arrival flows into the airport more efficiently, minimizing arrival meter fix congestion. This paper presents an analysis of air traffic flows across arrival meter fixes at Newark Liberty International Airport (EWR). Several scenarios of weather-impacted EWR arrival fix flows are described. Furthermore, multiple linear regression and regression tree ensemble learning approaches for translating sector Weather Impacted Traffic Indexes (WITI) to EWR arrival meter fix throughput are examined. These weather translation models are developed and validated using EWR arrival flight and weather data for the period of AprilSeptember in 2014. This study also compares the performance of the regression tree ensemble with traditional multiple linear regression models for estimating the weatherimpacted throughput at each of the EWR arrival meter fixes. For all meter fixes investigated, the results from the regression tree ensemble weather translation models show a stronger correlation between model outputs and observed meter fix throughput than that produced by multiple linear regression method.
\end{abstract}

Keywords-Air Traffic Control, Airport Meter Fix, Weather, Machine Learning Model.

\section{INTRODUCTION}

In today's airport arrival operations, as an aircraft transitions for landing, air traffic controllers guide the aircraft from cruise altitude to the runway. In order to ensure that aircraft are at appropriate altitudes, speeds, and separation, arrival aircraft are metered over the airport arrival meter fixes prior to entering the airport terminal area. It is crucial for air traffic controllers to manage airport arrival traffic to ensure efficiency and safety. Directing aircraft over a specified meter fix is required to maintain minimum safety separation, which would be increased during bad weather ${ }^{[1,2]}$. The reduced meter fix capacity can sometimes cause unnecessary arrival airborne delay and airborne holding; even through the airport capacity is still underutilized. Modeling the weatherimpacted meter fix flow has been conceived to assist controllers in determining the degree of arrival flow reduction caused by weather. In post operations analysis, the model can be used to check if the recorded operation was within the range of the control operations under similar circumstances.

Efforts have been made during the past decade to understand the connection between weather and capacity both at the airport and airspace level. Except for some research that uses controller workload related airspace complexity on sector capacity estimation, a common approach in many studies is to develop weather transition models using the historical distribution of throughput as targets during convective weather ${ }^{[3-11]}$. To date, there is little work on weather-impacted capacity investigation for non-standard airspace regions such as airport arrival meter fixes. To model the connection between weather and capacity (acceptance rate) for the region, one may also rely on the inspection of historical airport arrival meter fix throughput affected by the convective weather.

This paper provides a study of characteristics of arrival meter fix flow for Newark Liberty International Airport (EWR). It also describes several scenarios illustrating the weather impacts on the arrival meter fix flow. To model the weather effects on EWR arrival meter fix throughput, WITIs for multiple sectors around the meter fixes and terminal arrival routes were selected as the inputs for modeling the throughput. Multiple linear regression and regression tree ensemble learning approaches were used for the modeling. A comparison of these two methods for modeling each EWR arrival meter fix throughput is also presented in this paper. The assessment is conducted by using air traffic and weather data from April to September in 2014. 
Section II of this paper describes the characteristics of EWR arrival meter fix flow. Several scenarios of weatherassociated EWR meter fix throughput are detailed in section III. Multiple linear regression and regression tree ensemble modeling approaches and validation method are explained in Section IV. Section V shows the computational results for estimating EWR arrival meter fix throughput by various methods mentioned above. Finally, concluding remarks are in Section VI.

\section{EWR ARRIVAL METER FIX FLOW}

The air traffic controllers meter aircraft over arrival Meter Fixes (MF) to manage the traffic arrival flows prior to entering airport terminal airspace safely and efficiently. There are four MFs about 40 nautical miles from EWR: SHAFF at the north of EWR, PENNS at the west, and DYLIN and RBV at the south (see Fig. 1). The EWR meter fix ring was then defined as a ring with a $40 \mathrm{~nm}$ radius from the center at EWR. The arrival aircraft directions at the EWR MF ring to EWR (see Fig. 2) show that EWR arrivals were concentrated at the following three directions: from the south (ZDC center) across DYLIN (52 $2^{\circ}$ to EWR), from the east (ZOB center) across PENNS $\left(105^{\circ}\right)$, and from the north (ZBW center) across SHAFF $\left(161^{\circ}\right)$.

The EWR MF arrival throughput from the three directions was defined as the number of arrival aircraft passing through the MF ring with the directions to EWR within $\left(270-75^{\circ}\right),\left(75^{\circ}-135^{\circ}\right)$, and $\left(135^{\circ}-270^{\circ}\right)$ for DYLIN, PENNS, and SHAFF MF arrival flows, respectively. The calculated daily EWR MF throughput for April-September, 2014 was consistent with the ASPM (Aviation System Performance Metrics) daily arrival rates, such as the counts for ASPM efficiency computation, ASPM metrics, and Enhanced Traffic Management System (ETMS) arrivals.

Fig. 3 is a vertical stacked bar chart for the three EWR arrival MF hourly rates on July 29, 2014 (Tuesday) that is a typical good weather day. The EWR MF flow rates for SHAFF, PENNS, and DYLIN are displayed in blue, green, and yellow bars, respectively. In the figure, the total EWR MF flow rate, i.e. the sum of three MF flow rates, was consistent with the EWR OAG (Official Airline Guides) scheduled arrival rate (cyan line), even though the average flight time from MFs to the airport was about 15 minutes. The EWR OAG arrival rates and EWR total MF throughput were below the airport capacity, AAR (red line).

The arrival aircraft ground speeds and Flight Levels (FL) of EWR arrival aircraft at the MF ring for the three MFs are displayed in Fig. 4 and 5, respectively. The average ground speeds at the MF ring for three EWR MF flows were similar, about 300 knots. The aircraft FLs at the MF ring for the three EWR MF flows were consistent with those required by the standard terminal arrival route (STAR) for an altitude range of 7000-10000 ft. The hourly EWR arrival flow rates at the MF are shown in Fig. 6. Assuming a rate of 20 arrival aircraft per hour as EWR MF operational acceptance rate (capacity), the arrow displayed in the figure points to the $98^{\text {th }}, 99.7^{\text {th }}$, and $99^{\text {th }}$ percentiles for DYLIN, PENNS, and SHAFF throughput, respectively. Most MF throughput values showed in Fig. 6 were restricted by MF upstream demand and/or the EWR airport capacity. In case where one MF flow exceeded 20 aircraft per hour, it was usually because other MFs were blocked by bad weather (see scenarios discussed in subsection D of section III). Using the average ground speed of 300 knots at MF, the average allowed lateral separation between two aircraft in an hour was about 15 nautical miles for 20 aircraft.

In Fig. 7, the normalized flows, defined as the percentages of the MF flows divided by the total MF flow, for the three EWR MFs are displayed. Table 1 lists $25^{\text {th }}$, $50^{\text {th }}$ (medium), and $75^{\text {th }}$ percentiles of the normalized MF flow distributions. Even though the medium of DYLIN flow was higher than that for PENNS and SHAFF, they were all very close to one third of the total EWR MF flows. This indicates the EWR arrival flows were usually distributed evenly over three meter fixes under normal operation conditions. With a maximum EWR AAR of 48 aircraft per hour from ASPM for the year of 2014, even for the high demand cases, each MF throughput would not reach the capacity of 20 aircraft per hour.

During severe convective weather, one or more EWR arrival meter fixes could be partially or totally blocked by weather, therefore the arrival meter fix air traffic flows would have to be reduced. If the total EWR arrival demand was not scaled down accordingly, the other meter fix flows might have to be increased to reach or exceed the capacity of 20 aircraft per hour in order to minimize airborne delays and aircraft holding. To illustrate weather impacts on EWR arrival meter fix air traffic flows, several scenarios are presented in the next section.

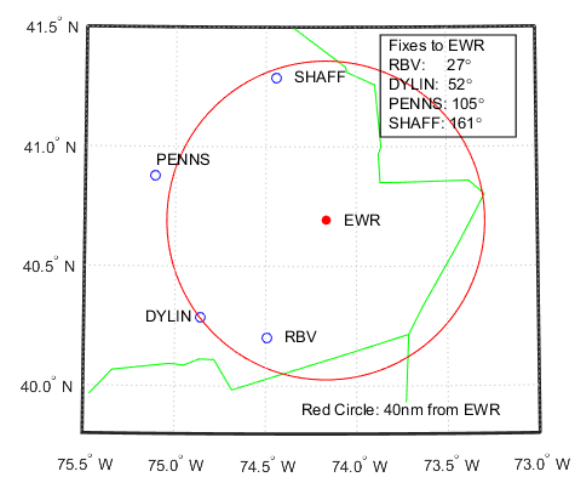

Fig. 1. EWR Arrival Meter Fix Positions 


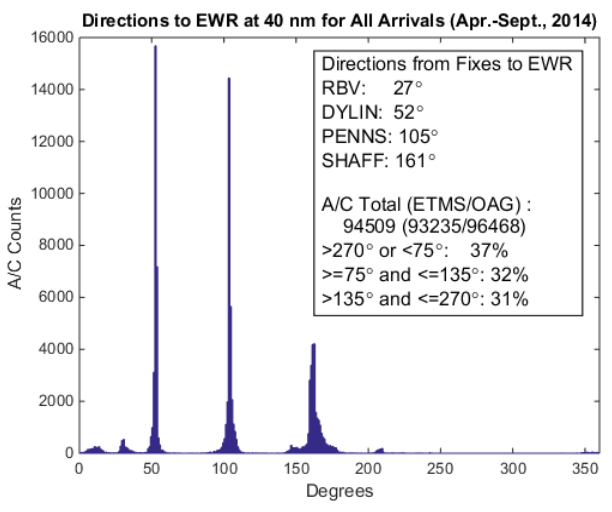

Fig. 2. Directions from Arrival Aircraft at $40 \mathrm{~nm}$ to EWR

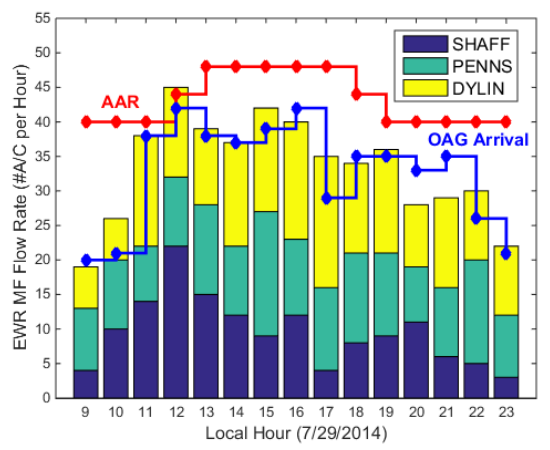

Fig. 3. EWR MF, OAG Arrival Rate, and AAR
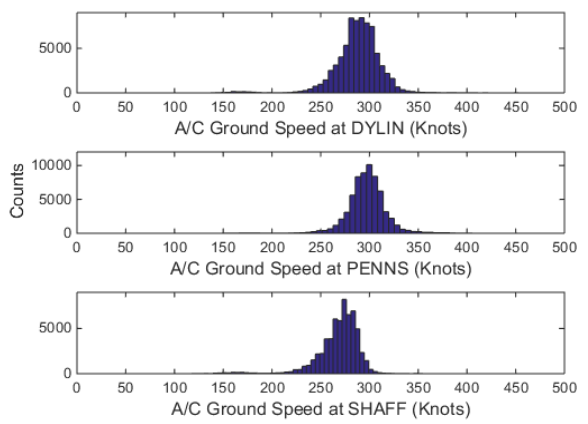

Fig. 4. EWR Arrival Aircraft Ground Speeds at MFs
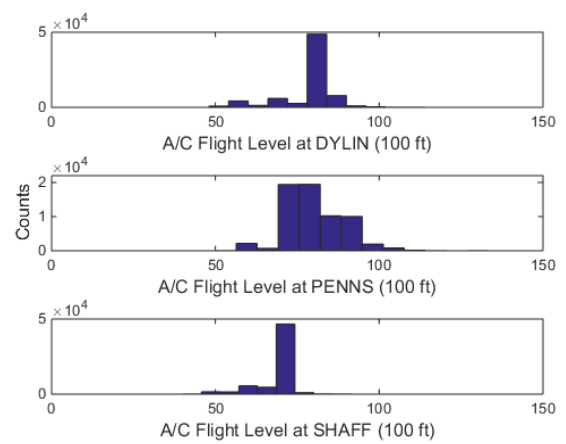

Fig. 5. EWR Arrival Flight Levels at MFs
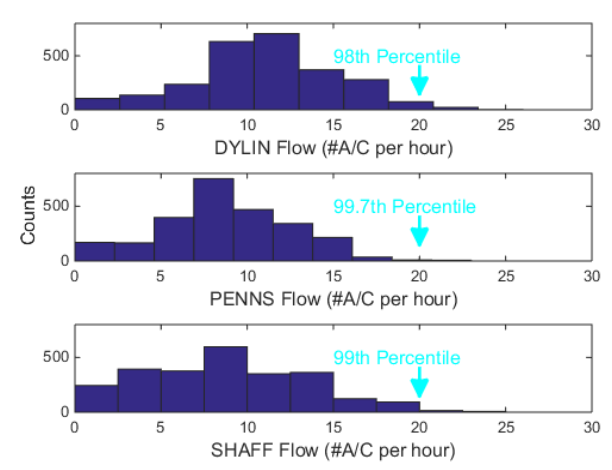

Fig. 6. EWR Arrival Hourly Flow Rate at MFs
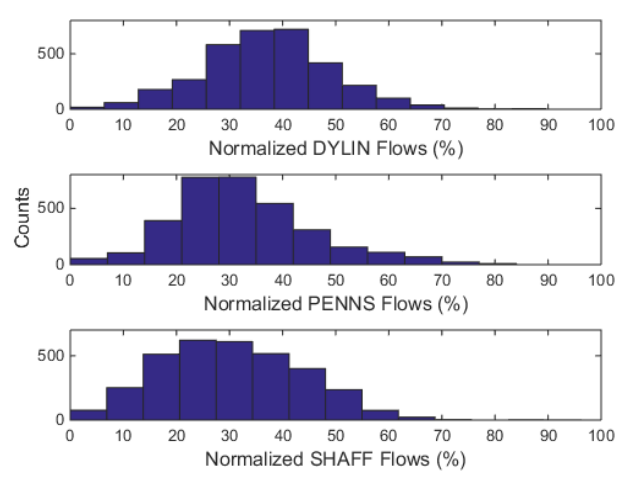

Fig. 7. EWR Arrival MF Normalized Flow Rates

Table 1. EWR MF Normalize Flow Distributions

\begin{tabular}{|c|c|c|c|}
\hline $\begin{array}{c}\text { MF } \\
\text { Normalized } \\
\text { flows }\end{array}$ & $\begin{array}{c}25^{\text {th }} \\
\text { Percentiles }\end{array}$ & $\begin{array}{c}50 \\
\text { Percentiles }\end{array}$ & $\begin{array}{c}75 \\
\text { Percentiles }\end{array}$ \\
\hline DYLIN & $29 \%$ & $37 \%$ & $44 \%$ \\
\hline PENNS & $24 \%$ & $31 \%$ & $39 \%$ \\
\hline SHAFF & $21 \%$ & $30 \%$ & $40 \%$ \\
\hline
\end{tabular}

\section{WEATHER INFLUENCE ON EWR ARRIVAL METER FIX FLOWS}

\section{A. EWR Arrival Flight Data and Capacity}

The positional data in the three-dimensional space (latitude, longitude, and altitude) for EWR arrival flights, including all minute-by-minute arrival flight tracks were selected from FAA Aircraft Situation Display to Industry (ASDI) data services. The ASDI information consists of components like flight plans, position reports, flight diversions, and cancellations. The position reports were the updates of aircraft positions every time when the computers were aware of a position amendment.

EWR airport arrival capacity, i.e. Airport Arrival Rate (AAR), is a dynamic parameter to specify the number of arrival aircraft that EWR airport can accept over the course of a given time interval (typically an hour or 15 
minutes). Observed airport hourly AAR data were collected from the FAA ASPM database. EWR demand, the number of aircraft that intended to land at EWR airport per hour and EWR hourly count of scheduled arrivals data were also collected from ASPM data for this analysis.

\section{B. Weather Data}

The main source of weather information for this study was the Corridor Integrated Weather System (CIWS), which was developed by MIT Lincoln Laboratory (MIT-LL) ${ }^{[12]}$. This weather product combines data from dozens of weather radars with satellite data, surface observations, and numerical weather models in order to improve the accuracy and timeliness of the storm severity information. It provides automated, real-time, high spatial resolution data at a 5-minute update rate, as well as threedimensional forecast of storms. CIWS also offers precipitation measured by vertically Integrated Liquid (VIL) and the Echo Tops forecast.

Based on the CIWS weather product MIT-LL has developed the Convective Weather Avoidance Model (CWAM) ${ }^{[13]}$. CWAM models the flight deviation behavior of pilots around severe weather as a function of reflectivity level and echo tops, and translates convective weather information from CIWS data into Weather Avoidance Fields (WAFs) at each flight altitude level. The WAF provides estimated probability of aircraft deviation around severe weather in en route airspace as a function of horizontal location. For each of the WAF files, the data include polygons of airspace regions where aircraft are likely to deviate around with corresponding avoidance probability thresholds, such as $80 \%$. This data is available for each of the flight levels from 25,000 $\mathrm{ft}$ (FL250) up to 45,000 $\mathrm{ft}$ (FL450) in 1,000 ft increments. The center and sector weather-impacted traffic indexes were calculated using CIWS and flight data.

\section{Weather Impacted Traffic Index (WITI) Model}

WITI indicates how "bad" the weather was based on the number of aircraft affected by it. Over the past decade, WITI, at an aggregated national and regional level, has been well accepted as an indication of National Airspace System (NAS) air traffic delays due to its strong correlation with the latter (for example, see [8]). In this study, the airspace hourly WITI is defined by using the flight and weather in the 4-dimentional space inside the airspace $S$ (typically a Center or sector) and time $H$ (typically an hour), as:

$$
W I T I=\sum_{(i, j, k, t) \in S, H} T_{i, j, k, t} W_{i, j, k, t}
$$

Here $i, j, k$, and $t$ are the latitude, longitude, altitude at a specific time $t$, respectively. $T$ is the number of reference flights with its track segment at $(i, j, k)$ and $t$ on the reference day. $W$ is assigned a value of 1 for the severe weather at a location $(i, j, k)$ and $t$, and a value of 0 otherwise. The reference days were identified as the days with no significant weather, but significant traffic demand and low NAS delays.

The normalized airspace hourly percentage WITI, $p W I T I$, is given as the division of

$$
p W I T I=W I T I / \sum_{(i, j, k, t) \in S, H} T_{i, j, k, t} .
$$

Thus the sector $p$ WITI values show how "bad" the air traffic affected by the weather within the sector in an hour.

\section{Scenarios of Weather Impact on MF throughput}

Two examples of bad weather days, July 8 and July 15 (Tuesdays) in 2014, are illustrated in Fig. 8. The vertical bar chart is used to show hourly ZNY (blue), ZBW (cyan), ZOB (green), and ZDC (yellow) center-level WITIs that change over hours. The figure shows there was inclement weather at ZOB on July 8 and bad weather at ZDC and ZNY on July 15. Air traffic controllers had to reduce the EWR arrival demands over these two days since the severe weather could have prevented EWR arrival aircraft from landing by blocking arrival MFs.

The EWR MF arrival flows on July 8 and 15 are displayed in Fig. 9 and 11, respectively. The vertical bar charts are used to show hourly flow rates of SHAFF, PENNS, and DYLIN meter fixes on those two days. The total EWR MF hourly throughput was below the scheduled hourly arrivals, even though the scheduled arrivals were similar in the comparison with those for the good weather day on July 29 in Fig. 3.

In Fig. 9, the total hourly MF throughput was much less than the scheduled arrival rates during 6:00-10:00 pm local time (EDT) due to decrease in the arrival PENNS flows (green bar). PENNS arrival throughput was totally blocked during the time period of 8:00-8:59 pm on July 8 . The EWR arrival flight tracks during 8:00-8:59 pm on July 8 are displayed in Fig. 10. The flight tracks across SHAFF and DYLIN meter fixes are shown as magenta and green lines, respectively. The CWAM weather contours at FL250 with $80 \%$ avoidance probability threshold at 8:00 pm on July 8 are displayed as red polygons in the figure. Since the severe weather was located just on the east side of PENNS, there were no flight tracks passing through PENNS in that hour. However, three flights from north (magenta lines) were flying through high WAFs and landed at EWR airport crossing through SHAFF. It's 
possible that these flights were actually flying over or under the high WAF, or penetrating low WAFs.

In Fig. 11, the total MF throughput was much less than the scheduled arrival rates during 11:00 am-7:00 pm on July 15 . The arrival MF DYLIN flow from south was totally blocked from 6:00 to 6:59 pm. The EWR arrival flights during that time period and CWAM weather contours at FL250 with $80 \%$ avoidance probability threshold at 6:00 pm on July 15 are shown in Fig. 12. The south arrival DYLIN flow was totally blocked by the severe weather at ZDC and ZNY as shown within red contours in Fig. 12. Since the FL of air traffic flows from PENNS and SHAFF MFs to the EWR runways were under FL100, the flows did not show weather disturbance, even though the area was covered by it at FL250.

To illustrate that one EWR meter fix flow could be pushed up over the capacity of 20 aircraft per hour due to the other MF blocked by weather, three scenarios are shown in Fig. 13-19. Fig. 13 shows that the EWR arrival flights were dramatically reduced during 1:00-9:00 pm time period on July 2 of 2014 when the widespread severe weather prevented landing at EWR airport. As the arriving aircraft approaches EWR, the plane had to slow down and was forced to enter a holding pattern. When the bad weather persisted, the holding aircraft had to find a way to land by rerouting to another MF if it was not diverted to an alternate airport. Diversions are very undesirable because of the large passenger delay and high cost to the airlines. The EWR arrival flight tracks during the time period of 4:00-4:59 pm and CWAM weather contours at 4:00 pm are revealed in Fig. 14. SHAFF and PENNS were partly blocked by the severe weather moving from the west to east, and almost all PENNS flows were rerouted to DYLIN. Some aircraft were forced to hold (seen as oval patterns) at various locations before being rerouted. During that hour, EWR arrival flights across DYLIN, PENNS, and SHAFF meter fixes were 26, 2, and 1, respectively.

A similar weather situation for the time period of 9:009:59 pm on August 31 is displayed in Fig. 15. However during that time, the DYLIN meter fix was totally blocked by the bad weather. All DYLIN flights were rerouted to PENNS. Some aircraft were forced to hold before rerouting. The EWR arrival flights across DYLIN, PENNS, and SHAFF meter fixes for the hour 9:00-9:59 pm were 0,21 , and 4 , respectively.

Fig. 16 shows EWR PENNS arrival fix flow was dramatically reduced from 5:00-7:59 pm on August 22 . The EWR arrival flights during the time period of 4:004:59, 5:00-5:59, and 7:00-7:59 pm with CWAM weather contours at 4:00, 5:00, and 7:00 pm on August 22 are displayed in Fig. 17, 18, and 19, respectively. During the time period of 4:00-8:00 pm, there was "popcorn"-like weather partially blocking flight route J-584 (see the yellow line) from the west to the east passing the PENNS as shown in the figures. In order to avoid the weather, almost all PENNS flows were rerouted to SHAFF starting from $5 \mathrm{pm}$ and lasting for three hours. EWR arrival flights across DYLIN, PENNS, and SHAFF meter fixes during the hour of 7:00-7:59 shown in Fig. 19 were 12, 1, and 24, respectively.

Some characteristics of the weather impact on EWR arrival meter fix flows can be summarized as follows:

- Widespread severe weather could prevent arrival aircraft from landing at the EWR airport by blocking the arrival meter fixes even though the airport capacity was underutilized. Strategic traffic flow managers and airlines would have to plan hours in advance to reduce the EWR arrival demand accordingly.

- The meter fix throughput was impacted by the inclement weather around the arrival meter fix and the standard terminal arrival routes.

- An individual EWR arrival meter fix throughput was affected not only by the total arrival meter fix flow but also by the aircraft rerouted from another meter fix due to weather.

- In some cases, the flight reroute pattern from one arrival meter fix to another had latency of a few hours even though the weather had been improved quite a lot.

- The total EWR meter fix throughput was constrained by the EWR upstream arrival demands and downstream airport capacity.

- A better understanding of airport meter fix capacity (acceptance rate) benchmark could be very useful for FAA and airline operations.
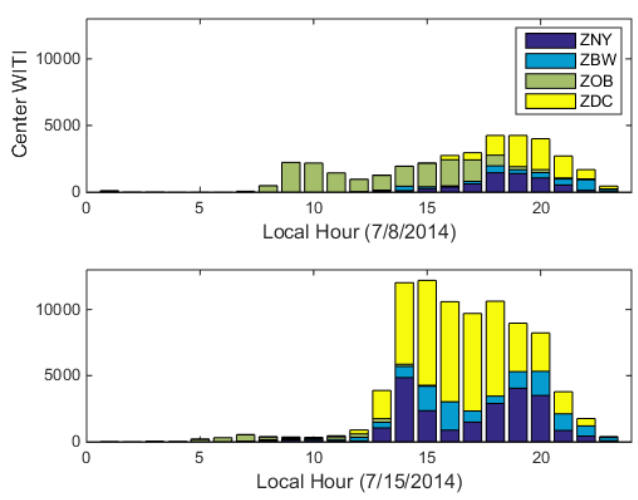

Fig. 8. Center WITIs on the Two Weather Days 


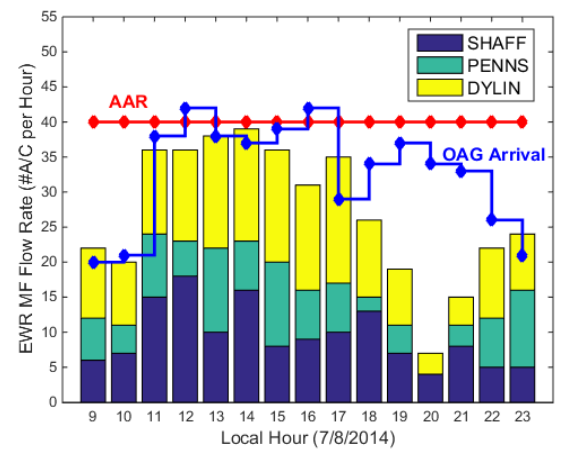

Fig. 9. EWR Arrival MF Flows on 7/8/2014

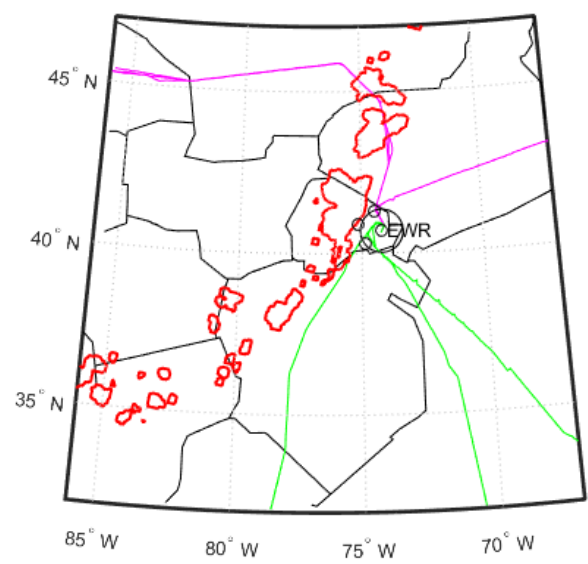

Fig. 10. EWR Arrival Flight during 8:00-8:59 pm and CWAM at 8:00 pm EDT on 7/8/2014

(Legend: Red polygons show CWAM weather at FL250 with $80 \%$ threshold. Green, blue, and magenta colored lines displays the flight tracks passing through DYLIN, PENNS, and SHAFF, respectively.)

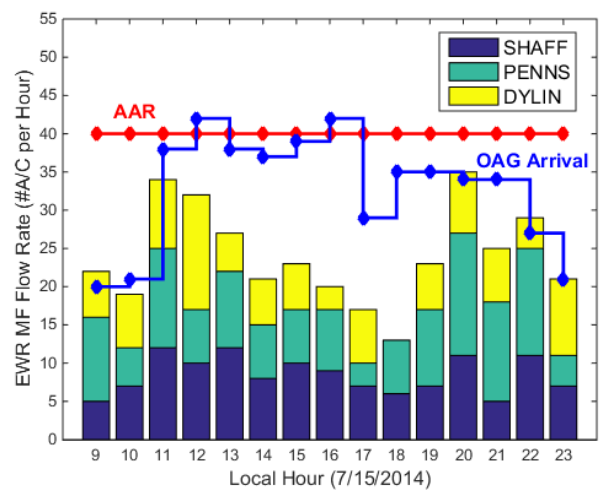

Fig. 11. EWR Arrival MF Flows on 7/15/2014

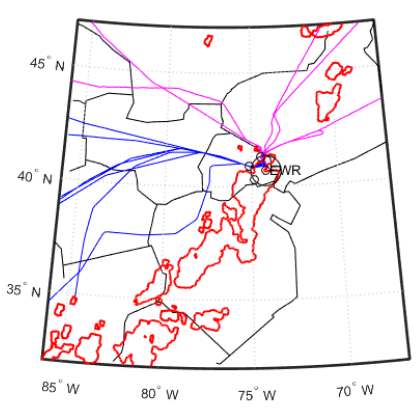

Fig. 12. EWR Arrival Flights during 6:00-6:59 pm EDT and CWAM at 6:00 pm EDT on 7/15/2014 (see Fig.10 Legend)

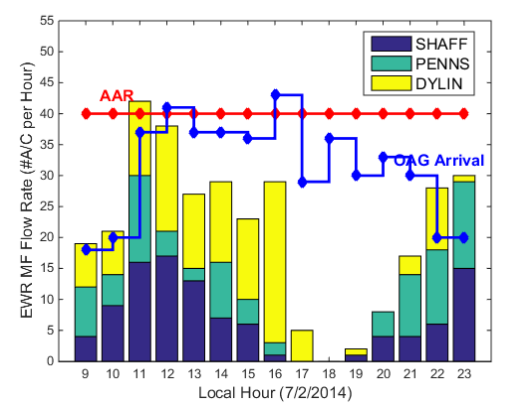

Fig. 13. EWR Arrival MF Flows on 7/2/2014

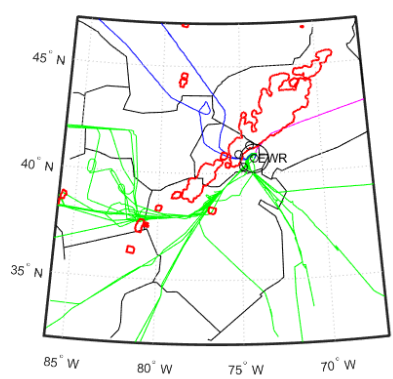

Fig. 14. EWR Arrival Flights during 4:00-4:59 pm EDT and CWAM at 4:00 pm EDT on 7/2/2014 (see Fig.10 Legend)

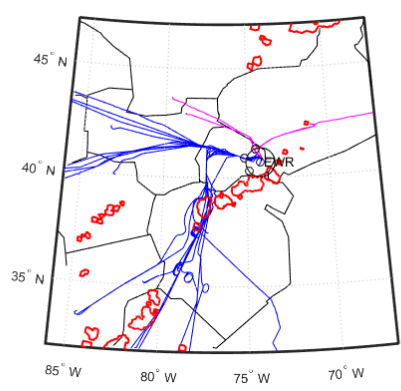

Fig. 15. EWR Arrival Flights during 9:00-9:59 pm EDT and CWAM at 9:00 pm EDT on 8/31/2014 (see Fig.10 Legend) 


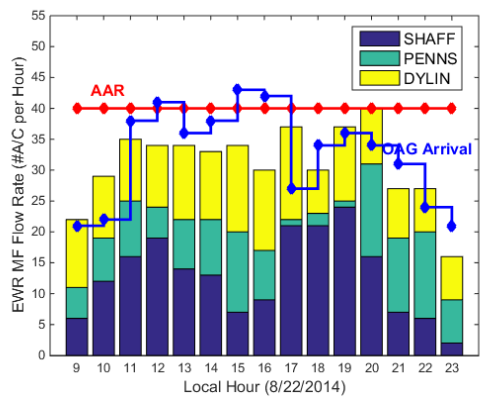

Fig. 16. EWR Arrival MF Flows on 8/22/2014

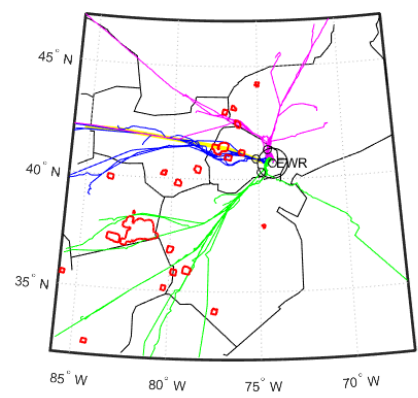

Fig. 17. EWR Arrival Flights during 4:00-4:59 pm EDT and CWAM at 4:00 pm EDT on 8/22/2014 (see Fig.10 Legend)

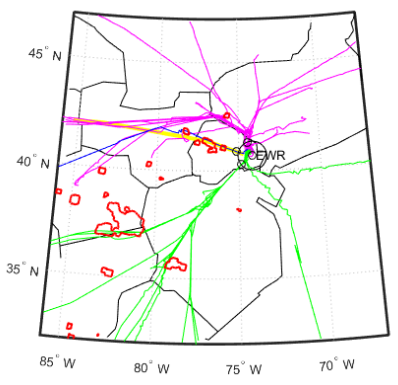

Fig. 18. EWR Arrival Flights during 5:00-5:59 pm EDT and CWAM at 5:00 pm EDT on 8/22/2014 (see Fig.10 Legend)

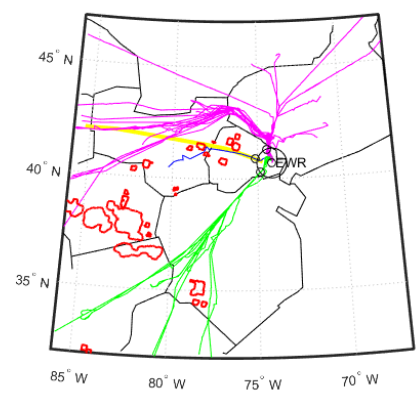

Fig. 19. EWR Arrival Flights during 7:00-7:59 pm EDT and CWAM at 7:00 pm EDT on 8/22/2014 (see Fig.10 Legend)

\section{E. Flight Reroute from one planned MF to the others}

It is clear that unforeseen weather influence on landings at EWR can lead to large delays and ultimately be very costly to the airlines and the travelling public. If the weather impacts are either short-lived or local, they can be mitigated effectively by using the available airspace. All the airborne and scheduled flights can be best handled using reroutes. The flights rerouted from one MF to the other MFs within a distance less than $400 \mathrm{~nm}$ between the reroute start point and EWR airport were selected from 2014 data. Almost all these reroutes were caused by weather. The flight rerouting percentage was calculated as the ratio between the number of rerouted flights and all flights. The results are listed in table 2 .

Table 2. Statistics of EWR Reroutes

\begin{tabular}{|c|c|c|}
\hline Planed MF & Reroute to MF & Percentages \\
\hline \multirow{2}{*}{ DYLIN } & PENNS & $1.06 \%$ \\
\cline { 2 - 3 } & SHAFF & $0.02 \%$ \\
\hline \multirow{2}{*}{ PENNS } & SHAFF & $1.39 \%$ \\
\cline { 2 - 3 } & DYLIN & $0.04 \%$ \\
\hline \multirow{2}{*}{ SHAFF } & PENNS & $0.05 \%$ \\
\cline { 2 - 3 } & DYLIN & $0.00 \%$ \\
\hline
\end{tabular}

It can be seen from Table 2 that rerouting happened more frequently from PENNS to SHAFF as well as from DYLIN to PENNS than that for the rest of the cases.

\section{F. Feature selection for the modeling}

To model hourly throughput of EWR arrival meter fixes altered by weather, events were selected if there was convective weather within a distance of $400 \mathrm{~nm}$ between the weather and EWR airport in a one hour time period during April-September, 2014. The hourly throughput observation for each EWR meter fix was selected as the model output, or target, and the hourly sector pWITIs selected were used as model inputs to indicate how "bad" the weather was. Based on the study of weather patterns from all scenarios (some were discussed above) and EWR terminal arrival routes, 49 sector pWITIs were chosen for modeling.

As concluded in previous subsection $\mathrm{D}$, the input variables also include hourly EWR arrival demand, EWR airport capacity AAR, and EWR meter fix flows from the previous hour.

\section{MODELING APPROACH}

Regression analysis was used to establish the relationships between weather (sector $p$ WITIs) and EWR arrival meter fix throughput. The techniques of Multiple Linear Regression (MLR) and Ensemble of Regression Tree (ERT) ${ }^{[14]}$ learning were applied for modeling the weather impact on EWR arrival MF throughput. 
Descriptions of these algorithms are provided in the following subsections.

\section{A. Multiple Linear Regression}

Multiple linear regression is an approach for modeling the relationship between more than one explanatory or independent input variable and a response or dependent variable using linear predictor functions whose unknown model parameters are derived from the observed training data. In Multiple Linear Regression, a real dependent variable $\delta$ is modeled as a linear function of multiple independent input variables $X_{1}, X_{2}, \ldots X_{\mathrm{n}}$, with model parameters $\alpha_{p}$ and $\beta$,

$$
\delta=\sum_{p=1}^{n} \alpha_{p} X_{p}+\beta
$$

In this paper, $\delta$ represents the EWR arrival MF throughput and $X_{\mathrm{p}}$ are the multiple predictor input variables, such as sector $p$ WITIs.

\section{B. Regression Tree Ensemble Learning}

ERT is a predictive model composed of a weighted combination of multiple regression trees using Machine Learning (ML) algorithms. ML is not a solution for every type of problem. There are certain cases where robust solutions can be developed without using ML techniques. For example, we could use the MLR models without any data-driven learning if MLR prediction of EWR meter fix throughput was in the accepted range comparable to the ERT algorithms.

The regression tree is a decision tree when the predicted outcome is a real number. A decision tree is built through a binary recursive partitioning process using a decision-tree algorithm. The tree is a flow-chart-like structure, where each internal node denotes a test of an input variable by the algorithm, each branch represents the outcome of a test, and each leaf or terminal node holds output variable values. A decision tree is a classic weak learner for which its predictive performance is better than random guessing and the training and prediction processes are fast by limiting the maximum depth of the tree.

Ensemble methods adopt multiple weak learners (regression trees) to obtain a better predictive performance than any of its individual constituent members can produce. Two popular and powerful machine learning ensemble methods, bagging ${ }^{[14]}$ and random forests ${ }^{[15]}$ were applied in this study.

Bagging stands for bootstrap aggregation. Ensemble Bootstrap Aggregation (EBA) was one of the first ensemble algorithms ever to be written, yet very effective. Bagging generates several Training Sets by using random sampling with replacement (bootstrap sampling), applies the regression tree algorithm to each data set, then takes the average among the models to calculate the predictions for the new data.

The ensemble random tree method, also known as Random Forests (ERF), is a variation of bagging. This method works by training multiple weak regression trees using a fixed number of randomly selected features (one third of the number of features), then takes the average value for the weak learners and assigns that value to the predictor. Typically, the number of weak trees generated could range from several hundreds to several thousands depending on the size and difficulty of the training set.

One advantage of the bagging and random trees methods is the relative ease with which the algorithms can be parallelized, which makes it a better selection for very large data sets. Both methods were implemented using MATLAB Treebagger function ${ }^{[16]}$.

\section{Validation of the Model}

A cross-validation approach was implemented for model validation. In cross-validation, a series of MLR or ERT (EBA and ERF) models were constructed, each time by dropping a different part of the data from the training set and applying the resulting model to predict the target. The merged series of predictions for dropped or test data were checked for accuracy against the observations. In one version of the cross-validation approach, called the group cross-validation approach, data are divided into $\mathrm{N}$ groups. A total of $\mathrm{N}$ models are then constructed with each using $\mathrm{N}-1$ data groups for model training, and the Nth one for testing. Tenfold cross-validation was used in this paper.

The linear Pearson's correlation coefficient $(r)$ is a measure of the strength of the association between the model estimations and observations on the test data. $r^{2}$ ranging from 0 to 1 indicates the absence to a perfect systematic association, respectively. Its value can be interpreted as the proportion of variance in observations that can be explained by the model estimations. $r$ and $r^{2}$ were used to compare the dependence between model estimation and observation in this paper.

Root Mean Squared Error (RMSE) was applied for assessment of algorithms. RMSE of EWR MF throughput estimates for $n$ test events is defined as

$$
R M S E=\sqrt{\frac{1}{n} \sum_{i=1}^{n}\left(y_{i}^{E}-y_{i}^{M}\right)} .
$$

Where $y_{i}^{E}$ and $y_{i}^{M}$ means the MF throughput model estimate and observation for the $i^{\text {th }}$ event, respectively. 


\section{COMPUTATIONAL RESULTS}

This section presents computational results and the comparisons between modeling techniques MLR and ERT (EBA and ERF). The EWR DYLIN, PENNS, and SHAFF meter fix throughput models were trained and tested by tenfold cross-validation using flight and weather data in the time frame of April-September of 2014.

The model performance comparisons for deriving DYLIN, PENNS, and SHAFF arrival MF throughput by the three methods are listed in Table 3, 4, and 5, respectively. The linear Pearson's correlation coefficient $(r)$, squared correlation coefficient $\left(r^{2}\right)$, and the Root Mean Squared Error (RMSE) for each method are displayed in the tables.

First, these tables reveal that the modeling by EBA and ERF machine-learning algorithms resulted in almost identical performance for estimating EWR arrival MF throughput using the features selected in this study.

It also shows the estimating performances of ERT (EBA and ERF) machine learning models were significantly better than that produced by the MLR model.

The model performance for EWR DYLIN arrival MF throughput estimates was better than that for either PENN or SHAFF. The possible explanation may be due to the fact that the DYLIN flow was the most dominant one (see Table 1) and the impact on DYLIN flow by rerouted aircraft from other MF is the smallest (see Table 2) among three direction EWR arrivals.

Table 3. Validation Results for DYLIN Throughput Modeling

\begin{tabular}{|c|c|c|c|}
\hline Method & $\boldsymbol{r}$ & $\boldsymbol{r}^{2}$ & $\boldsymbol{R M S E}$ \\
\hline MLR & 0.803 & 0.645 & 3.25 \\
\hline EBA & 0.860 & 0.740 & 2.78 \\
\hline ERF & 0.861 & 0.742 & 2.77 \\
\hline
\end{tabular}

Table 4. Validation Results for PENNS Throughput Modeling

\begin{tabular}{|c|c|c|c|}
\hline Method & $\boldsymbol{r}$ & $\boldsymbol{r}^{2}$ & $\boldsymbol{R M S E}$ \\
\hline MLR & 0.657 & 0.432 & 3.35 \\
\hline EBA & 0.765 & 0.585 & 2.86 \\
\hline ERF & 0.764 & 0.583 & 2.87 \\
\hline
\end{tabular}

Table 5. Validation Results for SHAFF Arrival Modeling

\begin{tabular}{|c|c|c|c|}
\hline Method & $\boldsymbol{r}$ & $\boldsymbol{r}^{2}$ & $\boldsymbol{R M S E}$ \\
\hline MLR & 0.681 & 0.463 & 3.74 \\
\hline EBA & 0.810 & 0.656 & 3.00 \\
\hline ERF & 0.810 & 0.656 & 3.00 \\
\hline
\end{tabular}

The scatter plots between the observed and ERT (using EBA) estimated EWR arrival MF throughput for DYLIN, PENNS, and SHAFF are displayed in Fig. 20, 21, and 22, respectively. The 5th, 95th percentile and median lines are also included in the figures.
To illustrate the ERT modeling results in these plots, let's assume that the throughput is predicted as 2 aircraft during a given hour; then in approximately $90 \%$ of cases, the real throughput values for DYLIN, PENNS, or SHAFF would fall between 1 and 4,0 and 3.5 , or 0.7 and 5.5 aircraft for that hour, respectively. Similarly, if the estimated throughput were 8 aircraft per hour, then the real throughput values would fall between 5 and 12, 2 and 12, or 3.4 and 11 aircraft/hour for DYLIN, PENNS, or SHAFF MF, respectively in $90 \%$ of cases.

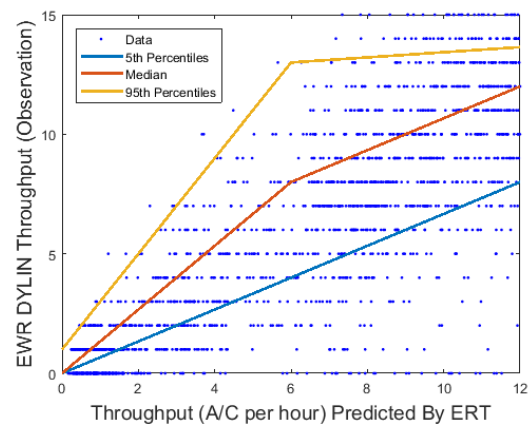

Fig. 20. DYLIN Throughput ERT Prediction Performance

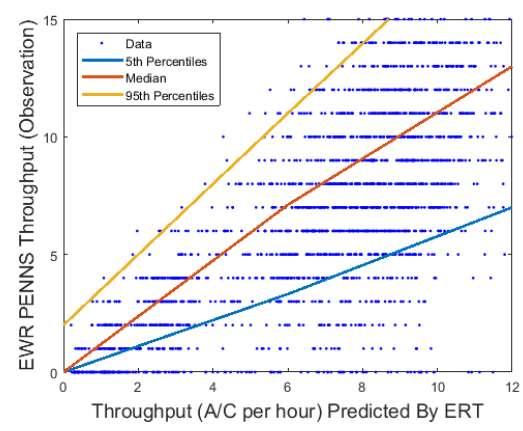

Fig. 21. PENNS Throughput ERT Prediction Performance

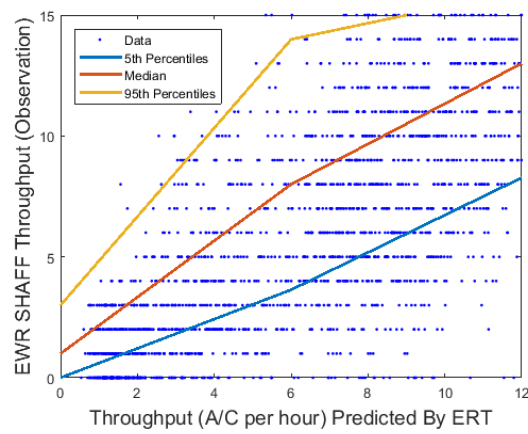

Fig. 22. SHAFF Throughput ERT Prediction Performance 


\section{CONCLUDING REMARKS}

This paper presents an extensive analysis of the EWR arrival meter fix flows over the period from April through September in 2014. The EWR arrival MF flows were passing through the MFs from the three directions. Factors affecting the flow at a given EWR arrival meter fix consist of convective weather, upstream airport arrival demand, downstream airport capacity, as well as the flows from other MFs. Widespread severe weather near to or over the MF could prevent arrival aircraft from landing at EWR airport even though the airport capacity is being underutilized. The analysis of several scenarios of weather-impacted EWR airport arrival meter fix flows and a summary of them are presented in subsection III.D. Multiple linear regression and two regression tree ensemble methods - bootstrap aggregation and random forest - were implemented using multiple sector $p$ WITIs to model each EWR arrival meter fix throughput.

EWR arrival meter fix throughput estimated by two regression tree ensemble approaches showed similar validation results. The estimated EWR MF throughput by the regression tree ensemble methods appeared to have a better correlation with actual observation than that from the multiple linear regression method. The regression tree ensemble estimations were accomplished by using supervised machine learning to train the models. All models were validated using a tenfold data cross validation method. For predicting the EWR meter fix throughput, the regression tree ensemble model was able to achieve the squared correlation coefficients of $0.74,0.58$, and 0.66 in comparison with $0.65,0.43$, and 0.46 by MLR for EWR meter fix DYLIN, PENNS and SHAFF throughput, respectively.

There are many opportunities to improve the meter fix models described in this paper. For example, the model of individual meter fix throughput under convective weather would be more useful if it captured the uncertainty in weather forecast, airport arrival demands, and airport capacity for 2-8 hour look-ahead time scales. Air traffic scheduling using the flight track based operations could be improved significantly if the superior performance of airport meter fix throughput models were incorporated.

\section{ACKNOWLEDGMENTS}

The author would like to thank Paul Lee and Deepak Kulkarni for their valuable advice and suggestions. Acknowledgment is also due to Heather Arneson and Shubha Ranjan who helped with flight and sector WITI data preprocessing.

\section{REFERENCES}

${ }^{1}$ Clifford, S.F., et al., "Weather Forecasting Accuracy for FAA Traffic Flow Management", the National Academies Press500 Fifth Street, N.W. Washington, D.C., 2003.

${ }^{2} \mathrm{http}$ //www.fly.faa.gov/Products/Training/Traffic_Ma nagement_for_Pilots/TFM_in_the_NAS_Booklet_ca10.pd $\mathrm{f}$ "Traffic Flow Management in the $\bar{N}$ National Airspace System" (Report). Federal Aviation Administration. October 2009.

${ }^{3}$ Callaham, M., DeArmon, J., Cooper, A., Goodfriend, J., Moch-Mooney, D., and Solomos, G., "Assessing NAS Performance: Normalizing for the Effects of Weather", $4^{\text {th }}$ USA/Europe ATM R\&D Seminar, Santa Fe, NM, Dec. 4-7, 2001.

${ }^{4}$ Chatterji, G. B., and Sridhar, B., "National Airspace System Delay Estimation Using Weather Weighted Traffic Counts", AIAA Guidance, Navigation and Control Conference, San Francisco, CA, August 15-18, 2004.

${ }^{5}$ Krozel, J., Mitchell, S., Polishchuk, V., and Prete, J., "Capacity Estimation for Airspaces with Convective Weather Constraints", AIAA Guidance, Navigation, and Control Conf., Hilton Head, SC, 2007.

${ }^{6}$ Klein, A., Cook, L., Wood, B., and Simenauer, D., "Airspace Capacity Estimation Using Flows and WeatherImpacted Traffic Index", Integrated Communications, Navigation and Surveillance Conference, 2008.

${ }^{7}$ DeLaura, R., and Evans, J., "An Exploratory Study of Modeling En route Pilot Convective Storm Flight Deviation Behavior", $13^{\text {th }}$ Conference on Aviation, Range and Aerospace Meteorology, New Orleans, LA, January 20-24, 2008.

${ }^{8}$ Sridhar, B., Wang, Y., Klein, A., and Jehlen, R., "Modeling Flight Delays and Cancellations at the National, Regional and Airport Levels in the United States", $8^{\text {th }}$ USA/Europe ATM R\&D Seminar, Napa, California, June 29 - July 2, 2009.

${ }^{9}$ Wang, Y. and Grabbe, S. "Comparison of Sector Capacity Weather Translation Models". NASA Tech Report TM-2010216401, 2010.

${ }^{10}$ Wang, Y and Sridhar, B. "Convective Weather Forecast Accuracy Analysis at Center and Sector Levels", Digital Avionics Systems Conference, 2012.

${ }^{11}$ Matthews, M.P., Veillette, M.S., Venuti, J.C., DeLaura, R.A. and Kuchar, J.K., "Heterogeneous Convective Weather Forecast Translation into Airspace Permeability with Prediction Intervals",Journal of Air Transportation, pp.41-54, 2016.

${ }^{12}$ Evans, J. and Ducot, E., "Corridor Integrated Weather System”, MIT Lincoln Laboratory Journal, Volume 16, Number 1, 2006.

${ }^{13}$ DeLaura, R., Robinson, M., Pawlak, M., and Evans, J., "Modeling Convective Weather Avoidance In En route Airspace”, MIT Lincoln Laboratory Project report NASA/A-6, June 2006.

${ }^{14}$ Breiman, L. Bagging Predictors. Machine Learning 26, pp. 123-140, 1996.

${ }^{15}$ Breiman, L. Random Forests. Machine Learning 45, pp. 5 32,2001

${ }^{16}$ MATLAB R2017a, Statistics Toolbox, TreeBagger Class, 2017.

36th Digital Avionics Systems Conference September 17-21, 2017 